\title{
Effects of age, BMI and sex on the glial cell marker TSPO - a multicentre $\left[{ }^{11} \mathrm{C}\right]$ PBR28 HRRT PET study
}

\author{
Jouni Tuisku ${ }^{1,2}$ (1) Pontus Plavén-Sigray ${ }^{3}$ • Edward C. Gaiser ${ }^{4,5} \cdot$ Laura Airas $^{1,6} \cdot$ Haidar Al-Abdulrasul ${ }^{1,6}$. \\ Anna Brück ${ }^{1,6}$ - Richard E. Carson ${ }^{4} \cdot$ Ming-Kai Chen $^{4} \cdot$ Kelly P. Cosgrove $^{4,5} \cdot$ Laura Ekblad $^{1} \cdot$ Irina Esterlis ${ }^{5}$. \\ Lars Farde $^{3,7}$ - Anton Forsberg ${ }^{3} \cdot$ Christer Halldin $^{3} \cdot$ Semi Helin $^{1} \cdot$ Eva Kosek $^{8} \cdot$ Mats Lekander $^{8,9} \cdot$ Noora Lindgren $^{1}$. \\ Päivi Marjamäki ${ }^{1}$ Eero Rissanen ${ }^{1,6}$ - Marcus Sucksdorff ${ }^{1,6}$ - Andrea Varrone ${ }^{3,9} \cdot$ HRRT $\left[{ }^{11} \mathrm{C}\right]$ PBR28 study group • \\ Karin Collste · Jean-Dominique Gallezot • Ansel Hillmer • Yiyun Huang • Caroline O. Höglund • Jarkko Johansson • \\ Aurelija Jucaite • Jon Lampa • Nabeel Nabulsi • Brian Pittman • Christine M. Sandiego • Per Stenkrona • Juha Rinne ${ }^{1,6}$. \\ David Matuskey $^{4,5} \cdot$ Simon Cervenka ${ }^{3}$
}

Received: 1 March 2019 / Accepted: 14 June 2019 / Published online: 30 July 2019

(C) The Author(s) 2019

\begin{abstract}
Purpose The purpose of this study was to investigate the effects of ageing, sex and body mass index (BMI) on translocator protein (TSPO) availability in healthy subjects using positron emission tomography (PET) and the radioligand $\left[{ }^{11} \mathrm{C}\right] \mathrm{PBR} 28$. Methods $\left[{ }^{11} \mathrm{C}\right] \mathrm{PBR} 28$ data from 140 healthy volunteers (72 males and 68 females; $\mathrm{N}=78$ with HAB and $\mathrm{N}=62$ MAB genotype; age range 19-80 years; BMI range 17.6-36.9) were acquired with High Resolution Research Tomograph at three centres: Karolinska Institutet $(\mathrm{N}=53)$, Turku PET centre $(\mathrm{N}=62)$ and Yale University PET Center $(\mathrm{N}=25)$. The total volume of distribution $\left(\mathrm{V}_{\mathrm{T}}\right)$ was estimated in global grey matter, frontal, temporal, occipital and parietal cortices, hippocampus and thalamus using multilinear analysis 1 . The effects of age, BMI and sex on TSPO availability were investigated using linear mixed effects model, with TSPO genotype and PET centre specified as random intercepts.

Results There were significant positive correlations between age and $\mathrm{V}_{\mathrm{T}}$ in the frontal and temporal cortex. BMI showed a significant negative correlation with $\mathrm{V}_{\mathrm{T}}$ in all regions. Additionally, significant differences between males and females were observed in all regions, with females showing higher $\mathrm{V}_{\mathrm{T}}$. A subgroup analysis revealed a positive correlation between $\mathrm{V}_{\mathrm{T}}$ and age in all regions in male subjects, whereas age showed no effect on TSPO levels in female subjects.

Conclusion These findings provide evidence that individual biological properties may contribute significantly to the high variation shown in TSPO binding estimates, and suggest that age, BMI and sex can be confounding factors in clinical studies.
\end{abstract}

Keywords PET $\cdot$ TSPO $\cdot\left[{ }^{11} \mathrm{C}\right] \mathrm{PBR} 28 \cdot$ Ageing $\cdot$ Sex difference $\cdot$ BMI

Members of the HRRT $\left[{ }^{11} \mathrm{C}\right] \mathrm{PBR} 28$ study group are listed in the Acknowledgments before References.

David Matuskey and Simon Cervenka share last author contributions.

This article is part of the Topical Collection on Neurology

Electronic supplementary material The online version of this article (https://doi.org/10.1007/s00259-019-04403-7) contains supplementary material, which is available to authorized users.

Jouni Tuisku

joupes@utu.fi

Extended author information available on the last page of the article

$\begin{array}{ll}\text { Abbreviations } \\ \text { AAL } & \text { Automated anatomical labeling } \\ \mathrm{B}_{\mathrm{max}} & \text { Total number of available binding sites } \\ \mathrm{BMI} & \text { Body mass index } \\ \mathrm{GM} & \text { Grey matter } \\ \mathrm{HAB} & \text { High-affinity binder } \\ \mathrm{HRRT} & \text { High resolution research tomograph } \\ \mathrm{kg} & \text { Kilogram } \\ \mathrm{K}_{\mathrm{d}} & \text { Radioligand affinity } \\ \mathrm{kDa} & \text { KiloDalton } \\ \mathrm{KI} & \text { Karolinska Institutet } \\ \mu \mathrm{g} & \text { Microgram } \\ \mathrm{MAB} & \text { Mixed-affinity binder } \\ \mathrm{MBq} & \text { MegaBecquerel }\end{array}$




$\begin{array}{ll}\text { MRI } & \text { Magnetic resonance imaging } \\ \text { nmol } & \text { Nanomole } \\ \text { PET } & \text { Positron emission tomography } \\ \text { ROI } & \text { Region of interest } \\ \text { sd } & \text { Standard deviation } \\ \text { SPM } & \text { Statistical parametric mapping } \\ \text { TPC } & \text { Turku PET Centre } \\ \text { TSPO } & \text { Translocator protein } \\ \text { V }_{T} & \text { Total distribution volume }\end{array}$

\section{Introduction}

The translocator protein (TSPO) is an $18 \mathrm{kDa}$ protein structure located on the outer mitochondrial membrane. In the brain, TSPO is expressed in microglia, astrocytes, endothelial and smooth muscle cells and even neurons (although in lower levels compared to tissues which are essential for steroid production or lipid storage and metabolism) [1,2]. Although TSPO is present throughout the brain under normal physiological conditions [3-5], in vitro studies have shown that TSPO expression increases in response to immune activation [6]. TSPO radioligands have therefore been used together with molecular imaging techniques to study brain immune activation.

A limitation of clinical TSPO PET studies is that TSPO shows high interindividual variability, even after accounting for the effect of the TSPO gene polymorphism [7, 8]. It is not fully known to what extent this variability is influenced by individual physiological properties, such as age, body mass index (BMI) and sex. TSPO is involved in the transport of cholesterol across the mitochondrial membrane, a requirement for steroid synthesis; additionally it is involved in immunomodulation as well as mitochondrial respiration and metabolism [9]. These processes are likely to be related to ageing, as well as hormonal steroid function [10]. However, previous in vivo human research using positron emission tomography (PET) imaging to investigate influences of age and sex on TSPO expression have been inconclusive. Studies have showed both higher TSPO levels with increasing age [11-14] and no effect [15-17]. Similarly, no effects of sex or BMI on TSPO expression were found in a recent TSPO study of healthy control subjects [13], whereas higher TSPO levels were observed in females in a clinical study with small sample size [18].

In vivo PET studies usually involve small sample sizes, and this low statistical power adds difficulty to detect significant effects. In order to more conclusively examine the effects of ageing, BMI and sex on TSPO availability in healthy individuals, we combined TSPO radioligand $\left[{ }^{11} \mathrm{C}\right] \mathrm{PBR} 28$ data acquired using a High Resolution Research Tomograph (HRRT) from three different institutes. This resulted in the largest TSPO PET sample to date of healthy control subjects imaged using the same radioligand and PET system.

\section{Materials and methods}

This study was based on $\left[{ }^{11} \mathrm{C}\right] \mathrm{PBR} 28$ data from 140 healthy volunteers that had previously been enrolled at three centres: Karolinska Institutet (KI; $\mathrm{N}=53$ ), Turku PET Centre (TPC; $\mathrm{N}=62$ ) and Yale University PET Center (Yale; $\mathrm{N}=25$ ). Descriptive statistics for age, BMI, sex and TSPO genotype for each centre are presented in Table 1. Ethical approvals were obtained from the Stockholm Regional Ethical Board, Hospital District of Southwest Finland and the Yale Human Investigation Committee, respectively, for KI, Turku and Yale data.

Exclusion criteria for participation at all centres were evidence of a current or previous major psychiatric illness (e.g., schizophrenia or major depression), alcohol or drug dependence, or history of a serious somatic or neurological illness. All subjects had a physical exam and medical history to confirm criteria. Subjects who had medication for diabetes mellitus ( $\mathrm{N}=7$, exclusively in Turku data) were excluded from the initial study sample of 147 individuals. Three subjects of Turku data were smoking regularly, and for several Turku data subjects the current smoking status was unknown $(\mathrm{N}=10)$. A
Table 1 Study subject characteristics of Karolinska Institutet (KI), Turku PET Centre (TPC) and Yale University PET Center (Yale) data

\begin{tabular}{llllll}
\hline Parameter & KI & TPC & Yale & Total & $p^{*}$ \\
\hline Number of subjects & 53 & 62 & 25 & 140 & - \\
HAB genotype subjects & 31 & 34 & 13 & 78 & - \\
MAB genotype subjects & 22 & 28 & 12 & 62 & - \\
Male / Female subjects & $30 / 23$ & $26 / 36$ & $16 / 9$ & $72 / 68$ & - \\
Age range & $20-72$ & $42-80$ & $19-55$ & $19-80$ & - \\
Age (mean \pm sd) & $44.3 \pm 17.3$ & $68.7 \pm 7.3$ & $33.4 \pm 11.6$ & $53.2 \pm 19.1$ & $p<0.001$ \\
BMI range & $17.6-30.5$ & $19.1-35.2$ & $20.4-36.9$ & $17.6-36.9$ & - \\
BMI (mean \pm sd) & $23.3 \pm 2.9$ & $26.1 \pm 3.7$ & $26.9 \pm 4.2$ & $25.2 \pm 3.8$ & p $<0.001$ \\
\hline
\end{tabular}

$H A B$ high-affinity binder, $M A B$ mixed-affinity binder

*The group differences in age and BMI were investigated with the one-way analysis of variance 
subset of Turku data subjects also used cholesterol-lowering statins ( $\mathrm{N}=11$; 3 males, 8 females; age 70.7 (3.6); BMI 26.8 (4.1)) and hormonal replacement medication $(\mathrm{N}=2)$. Prior to imaging, all participants were genotyped for the rs6971 polymorphism of the TSPO gene and categorized as mixed-affinity binders (MABs) or high-affinity binders (HABs) utilizing methods described previously [7, 19]. Low-affinity binders were excluded from this study.

Structural MR imaging (MRI) was performed to exclude individuals with anatomical abnormalities and to allow for the delineation of anatomical regions for the PET data analysis after coregistration. T1-weighted MRI scans were acquired using a $3 \mathrm{~T}$ scanner (Discovery MR750 system (GE, Milwaukee, WI, at KI; Philips Ingenuity TF PET/MR, Philips Medical Systems, Cleveland, OH, USA, at TPC; Trio system, Siemens Medical Solutions, Malvern, Pennsylvania, at Yale).

All PET examinations were performed on an HRRT scanner (Siemens/CTI, Knoxville, TN, USA). The $\left[{ }^{11} \mathrm{C}\right] \mathrm{PBR} 28$ synthesis in TPC is described in the online resource. In KI and Yale the $\left[{ }^{11} \mathrm{C}\right] \mathrm{PBR} 28$ was prepared as previously described $[19,20]$. The injection dose was administered as a rapid intravenous bolus, where the mean (sd) of injected activity, molar activity and injected mass at the time of injection, respectively, at each centre was 413.5 (53.4) MBq, 328.5 (148.0) $\mathrm{MBq} / \mathrm{nmol}$ and $0.009(0.004) \mu \mathrm{g} / \mathrm{kg}$ at KI; 494.3 (18.9) MBq, 286.9 (120.0) MBq/nmol and $0.012(0.012)$ $\mu \mathrm{g} / \mathrm{kg}$ at TPC; and $575.3(151.6) \mathrm{MBq}, 178.7(164.0) \mathrm{MBq} /$ $\mathrm{nmol}$ and $0.036(0.036) \mu \mathrm{g} / \mathrm{kg}$ at Yale. Data were acquired while participants were at rest over 70,75 or $90 \mathrm{~min}$, respectively at TPC, KI and Yale. The present analysis was restricted to $70 \mathrm{~min}$ at TPC, and $75 \mathrm{~min}$ at KI and Yale. The further details of PET data acquisition, reconstruction and frame lengths, as well as arterial blood sampling and radiometabolite analysis are presented in the online resource.

All 4D PET data were preprocessed at each centre in a similar manner, in which PET images were realigned and coregistered to anatomical MR images using SPM12 software (Wellcome Trust Centre for Neuroimaging, London, UK) running in MATLAB (The Mathworks, Natick, MA), or Bioimage Suite (version 2.5; http:// www.bioimagesuite.com). The MR images were further segmented into tissue classes using SPM. The grey matter segment was thresholded $(\mathrm{GM}>0.5)$ and then used as the main region of interest for the statistical analysis. Additionally, six automated ROIs (frontal, temporal, occipital and parietal cortices, hippocampus and thalamus) were generated by using the Automated Anatomical Labeling (AAL) template [21]. These AAL ROIs were first coregistered to summed PET images via the deformation fields obtained from the MRI segmentation and then multiplied with the thresholded GM segment, before the ROI extraction.
The total volume of distribution $\left(\mathrm{V}_{\mathrm{T}}\right)$ was estimated at each centre using multilinear analysis $1[22]\left(t^{*}=30 \mathrm{~min}\right)$ with the metabolite and the delay corrected arterial plasma curves as an input function. Uniform weights were used in the MA1 analysis at KI and Turku, whereas at Yale the weights were based on the noise equivalent counts in each frame. The normality of the regional $\mathrm{V}_{\mathrm{T}}$ estimates were inspected with Shapiro-Wilk test of normality and the Q$\mathrm{Q}$ plot. The main research question of this study was to examine the associations between $\left[{ }^{11} \mathrm{C}\right] \mathrm{PBR} 28$ binding and age, sex as well as BMI. This was done using a linear mixed effects model, with TSPO genotype and PET centre specified as random effects, allowing their intercepts to vary freely. The results from this model are reported as "confirmatory", as the associations were hypothesized $a$ priori. Having observed the results from the confirmatory analysis, we performed a set of follow-up tests, examining males and females separately, as well as sex-age interaction and centre effects. The results of these analyses are reported as "exploratory", as they are all post-hoc. The results are presented as unstandardized regression effect estimates with $95 \%$ confidence intervals. All statistical analyses were conducted using lme4 and lmerTest packages in R (version 3.5.0 "Joy in Playing"). Previous studies have shown high correlations between $\left[{ }^{11} \mathrm{C}\right] \mathrm{PBR} 28 \mathrm{~V}_{\mathrm{T}}$ estimates in different ROIs [23] and in this dataset all inter-regional Pearson's correlations were $>0.96$ (all $p<10^{-15}$ ). Hence, we considered the ROI analyses to be highly dependent comparisons and set the alpha threshold to 0.05 (two-tailed). The main research questions, outcome measures and overall statistical approach were all determined before data was shared and analysed, in a signed and dated Memorandum of Understanding (available upon request).

Availability of data and material The data will be available upon request from the principal investigators, as allowed by legislation on data integrity in the country of each participating centre.

\section{Results}

There were significant differences in the age and BMI between centres (Table 1), but there were no significant differences in these variables between HABs and MABs, or between males and females (Table 2). Figure 1 illustrates the relationships of age, BMI and sex to grey matter $\left[{ }^{11} \mathrm{C}\right]$ PBR28 $\mathrm{V}_{\mathrm{T}}$. The Shapiro-Wilk test and the Q-Q plot indicated that the residuals of the statistical model were not normally distributed (see online Resource). Hence, all $\mathrm{V}_{\mathrm{T}}$ values were log-transformed prior to being inserted into the linear-mixed effect model, which then fulfilled 
Table 2 Study sample characteristics grouped by TSPO genotype (A) and sex (B)

\begin{tabular}{lllll}
\hline Group & Demographic & HAB & MAB & $p^{*}$ \\
(A) & Number & 78 & 62 & - \\
& Males/Females (n) & $35 / 43$ & $37 / 25$ & - \\
& Age (mean \pm sd) & $53.2 \pm 18.9$ & $53.1 \pm 19.6$ & 0.969 \\
& BMI (mean \pm sd) & $24.9 \pm 4.2$ & $25.5 \pm 3.1$ & 0.394 \\
Group & Female & Male & $p^{*}$ & \\
(B) & 68 & 72 & - & \\
& - & - & - & \\
& $54.0 \pm 19.6$ & $52.4 \pm 18.8$ & 0.632 & \\
& $24.7(4.3)$ & $25.6 \pm 3.3$ & 0.195 & \\
\hline
\end{tabular}

$H A B$ high-affinity binder, $M A B$ mixed-affinity binder

*The group differences were investigated with the Student's t test

the assumption of normally distributed residuals. Below, the results from log-transformed $\mathrm{V}_{\mathrm{T}}$ values are presented.

\section{Confirmatory results}

The regional fixed effect estimates with confidence intervals and $p$-values for the association between $\left[{ }^{11} \mathrm{C}\right] \mathrm{PBR} 28 \mathrm{~V}_{\mathrm{T}}$ and age, BMI and sex are presented in Table 3. There was a significant positive association between age and log-transformed $\mathrm{V}_{\mathrm{T}}$ in frontal and temporal cortex, but not in any other regions. Higher BMI levels were associated with lower logtransformed $V_{\mathrm{T}}$ in all regions. Additionally, significant differences between males and females were observed in all regions, with females showing higher $\left[{ }^{11} \mathrm{C}\right] \mathrm{PBR} 28$ binding. For example, an increase of one year of age predicted a $0.4 \%$ increase $\left(100 *\left(\mathrm{e}^{0.004}-1\right)=0.4\right)$ in temporal cortex $\mathrm{V}_{\mathrm{T}}$. Correspondingly, an increase of one BMI unit predicted a $2.4 \%$ decrease $\left(100 *\left(\mathrm{e}^{0.024}-1\right)=-2.4\right)$ in temporal cortex $\mathrm{V}_{\mathrm{T}}$. For females, temporal cortex $\mathrm{V}_{\mathrm{T}}$ was $16.3 \%$ higher $\left(100 *\left(\mathrm{e}^{0.151}-1\right)=16.3\right)$ compared to males.
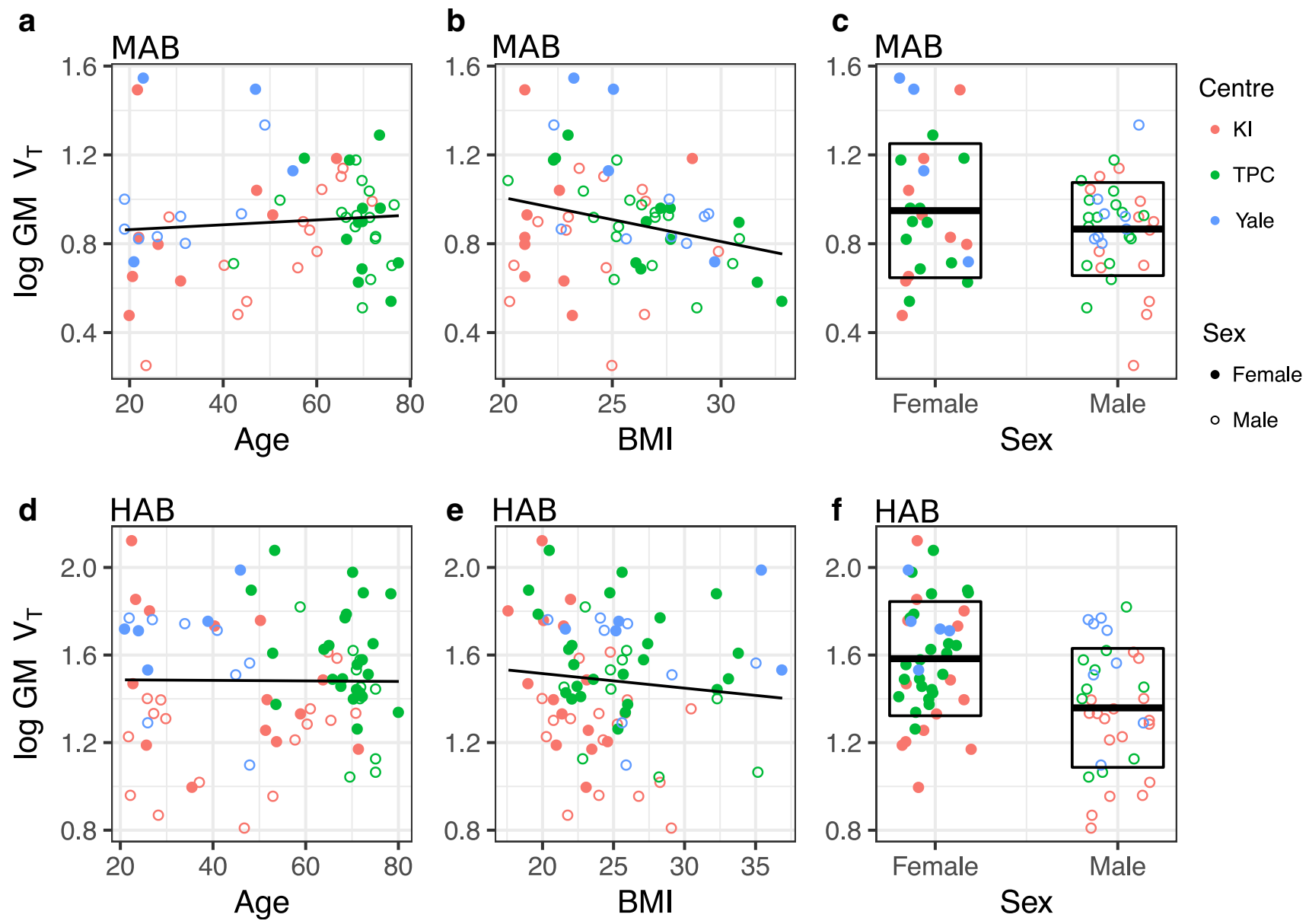

Fig. 1 The relationships of age, BMI and sex to log-transformed grey matter $\left[{ }^{11} \mathrm{C}\right] \mathrm{PBR} 28 \mathrm{~V}_{\mathrm{T}}$. Age vs. $\left[{ }^{11} \mathrm{C}\right] \mathrm{PBR} 28 \mathrm{GM} \mathrm{V}_{\mathrm{T}}$ in MAB genotype (A) and HAB genotype (B). BMI vs. $\left[{ }^{11} \mathrm{C}\right] \mathrm{PBR} 28 \mathrm{GM} \mathrm{V} \mathrm{V}_{\mathrm{T}}$ in MAB

$\left[{ }^{11} \mathrm{C}\right]$ PBR28 GM $\mathrm{V}_{\mathrm{T}}$ in MAB genotype subjects $(\mathbf{E})$ and HAB genotype subjects $(\mathbf{F})$. Box-plots in $(\mathbf{E})$ and $(\mathbf{F})$ represent the data means and standard deviations for both sexes 
Table 3 Fixed effects estimates with 95\% confidence intervals and p-values for age, BMI and sex as regressors for regional $\left[{ }^{11} \mathrm{C}\right] \mathrm{PBR} 28 \mathrm{log}-$ transformed $\mathrm{V}_{\mathrm{T}}$. $N=140$ for all analyses

\begin{tabular}{llll}
\hline Region & Age $(\beta,[\mathrm{CI}], \mathrm{p})$ & BMI $(\beta,[\mathrm{CI}], \mathrm{p})$ & $\operatorname{Sex}(\beta,[\mathrm{CI}], \mathrm{p})^{*}$ \\
\hline Gray matter & $0.003,[-0.001,0.007], p=0.080$ & $-0.022,[-0.034,-0.010], p<0.001$ & $0.155,[0.075,0.235], p<0.001$ \\
Frontal cortex & $0.004,[0.000,0.008], p=0.034$ & $-0.021,[-0.033,-0.009], \mathrm{p}=0.001$ & $0.164,[0.082,0.246], \mathrm{p}<0.001$ \\
Temporal cortex & $0.004,[0.000,0.008], \mathrm{p}=0.030$ & $-0.024,[-0.036,-0.012], \mathrm{p}<0.001$ & $0.151,[0.069,0.233], \mathrm{p}<0.001$ \\
Occipital cortex & $0.002,[-0.002,0.006], p=0.167$ & $-0.019,[-0.031,-0.007], \mathrm{p}=0.001$ & $0.161,[0.081,0.241], \mathrm{p}<0.001$ \\
Parietal cortex & $0.003,[-0.001,0.007], p=0.086$ & $-0.017,[-0.029,-0.005], \mathrm{p}=0.004$ & $0.172,[0.092,0.252], \mathrm{p}<0.001$ \\
Hippocampus & $0.002,[-0.002,0.006], p=0.349$ & $-0.026,[-0.038,-0.014], \mathrm{p}<0.001$ & $0.140,[0.054,0.226], \mathrm{p}=0.002$ \\
Thalamus & $0.003,[-0.001,0.007], p=0.067$ & $-0.023,[-0.035,-0.011], \mathrm{p}<0.001$ & $0.139,[0.051,0.227], p=0.002$
\end{tabular}

*Coding: Males $=0$, Females $=1$

Using the raw, untransformed $\mathrm{V}_{\mathrm{T}}$ values the results were similar to those presented above, except for the $\mathrm{V}_{\mathrm{T}}$-age association that was not statistically significant (see online resource).

\section{Exploratory results}

Due to the sex differences in $\mathrm{V}_{\mathrm{T}}$, the data were divided into two subgroups for studying the effects of age and BMI separately for both sexes. This subgroup analysis showed a significant positive association between age and logtransformed $\mathrm{V}_{\mathrm{T}}$ in all regions in male subjects, but not in female subjects (Table 4). There was also a significant negative association between BMI and $\left[{ }^{11} \mathrm{C}\right]$ PBR2 8 binding in males in all regions, but for females the association was significant only in the temporal cortex, hippocampus and thalamus. For example, an increase of one year of age predicted a $0.7 \%$ and a $0.1 \%$ increase in temporal cortex $\mathrm{V}_{\mathrm{T}}$, respectively, for males and females. Correspondingly, an increase of one BMI unit predicted $2.6 \%$ and $1.9 \%$ decrease in temporal cortex $\mathrm{V}_{\mathrm{T}}$, respectively, for males and females.
The interaction between age and sex was confirmed by adding an interaction term to the main effects model (fixed effects estimates are presented in the online resource). This analysis revealed a significant age and sex interaction in all regions except the occipital cortex and hippocampus. The fixed effect estimates of the association between age and $\log$-transformed $\mathrm{V}_{\mathrm{T}}$ in the grey matter for males and females are illustrated in Fig. 2.

\section{Robustness test}

To examine the robustness of our results, we performed the main analysis also by excluding the subjects who had cholesterol medication $(\mathrm{N}=11)$, hormone replacement therapy $(\mathrm{N}=2)$ and who were current smokers $(\mathrm{N}=3)$ (presented in the online resource; 16 excluded subjects, thus resulting in a total number of subjects of 124). The results were similar compared to the main analysis without exclusions.

Because of the of the significant differences in plasma parent fractions, as well as age and BMI distributions between centres, it can't be excluded that the overall age or

Table 4 Fixed effects estimates with 95\% confidence intervals and $p$-values for age and BMI as regressors for regional $\left[{ }^{11} \mathrm{C}\right] \mathrm{PBR} 28$ log-transformed $\mathrm{V}_{\mathrm{T}}$ in a separate subgroup analysis for males $(\mathrm{N}=72)$ and females $(\mathrm{N}=68)$

\begin{tabular}{llccc}
\hline Region & Age $(\beta,[\mathrm{CI}], \mathrm{p})$, Male & Age $(\beta,[\mathrm{CI}], \mathrm{p})$, Female & BMI $(\beta,[\mathrm{CI}], \mathrm{p})$, Male & BMI $(\beta,[\mathrm{CI}], \mathrm{p}), \mathrm{Female}$ \\
\hline \multirow{2}{*}{ Grey matter } & $0.006,[0.002,0.010]$, & $-0.002,[-0.008,0.004]$, & $-0.026,[-0.042,-0.010]$, & $-0.017,[-0.035,0.001]$, \\
& $\mathrm{p}=0.002$ & $p=0.542$ & $p=0.002$ & $p=0.061$ \\
Frontal cortex & $0.007,[0.003,0.011]$, & $-0.001,[-0.007,0.005]$, & $-0.024,[-0.040,-0.008]$, & $-0.015,[-0.033,0.003]$, \\
& $\mathrm{p}=0.001$ & $p=0.645$ & $p=0.004$ & $p=0.085$ \\
Temporal & $0.007,[0.003,0.011]$, & $-0.001,[-0.007,0.005]$, & $-0.026,[-0.042,-0.010]$, & $-0.019,[-0.037,-0.001]$, \\
cortex & $p=0.001$ & $p=0.745$ & $\mathrm{p}=0.002$ & $p=0.039$ \\
Occipital cortex & $0.005,[0.001,0.009]$, & $-0.002,[-0.008,0.004]$, & $-0.023,[-0.039,-0.007]$, & $-0.013,[-0.031,0.005]$, \\
& $p=0.006$ & $p=0.462$ & $\mathrm{p}=0.004$ & $p=0.123$ \\
Parietal cortex & $0.006,[0.002,0.010]$, & $-0.002,[-0.008,0.004]$, & $-0.021,[-0.037,-0.005]$, & $-0.012,[-0.030,0.006]$, \\
& $\mathrm{p}=0.002$ & $p=0.467$ & $p=0.010$ & $p=0.175$ \\
Hippocampus & $0.004,[0.000,0.008]$, & $-0.002,[-0.006,0.002]$, & $-0.027,[-0.045,-0.009]$, & $-0.020,[-0.036,-0.004]$, \\
& $p=0.046$ & $p=0.399$ & $p=0.005$ & $p=0.020$ \\
Thalamus & $0.006,[0.002,0.010]$, & $-0.001,[-0.005,0.003]$, & $-0.022,[-0.040,-0.004]$, & $-0.020,[-0.038,-0.002]$, \\
& $p=0.007$ & $p=0.755$ & $p=0.011$ & $p=0.030$
\end{tabular}




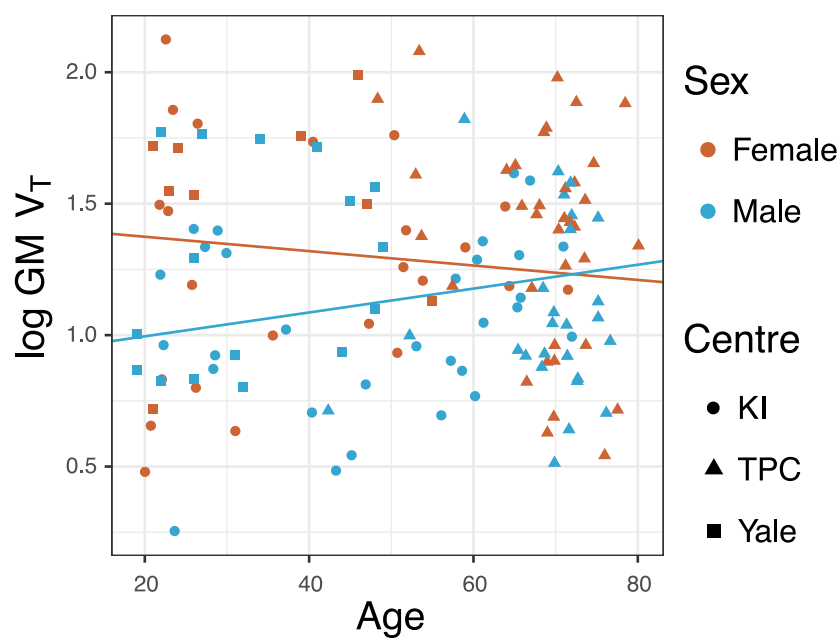

Fig. 2 The relationship between $\left[{ }^{11} \mathrm{C}\right] \mathrm{PBR} 28 \log$-transformed $\mathrm{V}_{\mathrm{T}}$ and age for males and females in the grey matter. The regression lines denote the fixed effects from two linear mixed effects models including either females or males, with TSPO genotype and PET center specified as random intercepts

BMI effects are attributed to only one centre. Therefore, a possible centre effect was studied in grey matter by inserting centre as a dummy predictor and as an interaction-effect to age, BMI and sex. The results were comparable to the main analysis. The age interaction estimate for Turku data was statistically significant, whereas the other interaction estimates were not significant (the results are presented in the online resource).

\section{Discussion}

In this retrospective multicentre collaboration study, constituting the largest harmonized TSPO PET sample to date, the results indicate that higher $\left[{ }^{11} \mathrm{C}\right] \mathrm{PBR} 28 \mathrm{~V}_{\mathrm{T}}$ is associated with lower BMI. Higher $\mathrm{V}_{\mathrm{T}}$ was found in females, as compared to males. Higher age was significantly associated to higher $\mathrm{V}_{\mathrm{T}}$ in frontal and temporal cortices, partly replicating a previous $\left[{ }^{11} \mathrm{C}\right] \mathrm{PBR} 28$ study [13]. A post hoc analysis however revealed that this association was present only in male subjects. These findings provide evidence that individual biological factors contribute significantly to the high variability seen in $\mathrm{V}_{\mathrm{T}}$ estimates, and suggest that age, BMI and sex can be confounding factors in clinical designs.

It has been suggested that with increasing age, microglia activate slower and exhibit less "surveying" morphology [24]. Several post-mortem human studies have indicated age-related alterations in microglia morphology and function as well as age-associated increases in total number of activated pro-inflammatory microglia phenotype [25-28]. Ageing has also been shown to be associated with increases in peripheral markers of inflammation [29-32]. In contrast, previous PET studies on brain TSPO in relation to age have been inconclusive [11-17, ]. However, the sample sizes have generally been small, limiting the conclusions that can be drawn. By using a several-fold larger dataset, a second-generation TSPO radioligand and full arterial sampling, our observation of a positive effect of age on $\left[{ }^{11} \mathrm{C}\right] \mathrm{PBR} 28$ binding in the frontal and temporal cortices is congruent with some of the previous studies, where positive correlations between age and TSPO binding were found in the thalamus [11], whole cortical GM [14] and in several cortical and subcortical areas $[12,13]$. Importantly, the present results were driven solely by the males, which could explain the conflicting results of no observed age effect found in other studies [15-17], where mixed samples of males and females were included.

Previous preclinical studies have shown also sex-related differences in microglial function [, 33]. These findings are supported by our observation of females showing higher $\left[{ }^{11} \mathrm{C}\right]$ PBR28 $\mathrm{V}_{\mathrm{T}}$ compared to males. Also, the potential link between TSPO and steroidogenesis could partly explain the association between immune function and sex steroid hormones [34]. Particularly, glial cells express receptors for estrogens and androgens, suggesting that there is an interplay between sex steroid hormones and the neuroinflammatory response [35-37]. As a consequence, an age-related change in both androgens and female sex hormones could explain the observed interaction of age and sex in $\left[{ }^{11} \mathrm{C}\right]$ PBR28 binding, where male TSPO levels increase with age, eventually reaching the higher TSPO level of females. Our finding of age-related sex difference in $\left[{ }^{11} \mathrm{C}\right] \mathrm{PBR} 28 \mathrm{~V}_{\mathrm{T}}$ may also provide a clue to the observed sex-specific differences of neuroinflammatory autoimmune diseases such as multiple sclerosis, which are more prevalent in younger females than in males [38].

While the immunostaining assays of a preclinical model of obesity have suggested that high-fat diet induced obesity increases neuroinflammation in the hypothalamus and hippocampus [39-42], in contrast we observed decreased $\left[{ }^{11} \mathrm{C}\right]$ PBR28 $\mathrm{V}_{\mathrm{T}}$ with higher BMI. A possible explanation for this could be that the peripheral TSPO expression is dysregulated in obese subjects, and that this effect is reflected also in the brain. Interestingly, a recent in vitro study on adipocytes, which are considered to be a major regulatory cell in obesity, suggested that TSPO expression is essential for the maintenance of the healthy adipocyte functions, and that TSPO activation in adipocytes improves their metabolic status in regulating glucose homeostasis [43]. On the other hand, a dysregulation of TSPO expression has been reported previously in a preclinical study, where the total number of available TSPO binding sites $\left(\mathrm{B}_{\max }\right)$ was significantly decreased in brown adipose tissue cells of obese mice compared to the lean controls, whereas there was no difference in the affinity of the 
ligand $\left(\mathrm{K}_{\mathrm{d}}\right)$ [44]. Although our results support an involvement of TSPO in obesity, we cannot determine if this effect is related to brain TSPO expression specifically or if this is reflective of systemic differences. For instance, TSPO levels in brain and peripheral blood cells were previously shown to correlate highly [45]. To our knowledge, the relationship between TPSO binding in brain and peripheral tissue (including adipose tissue) has not been investigated, and future studies on this topic are warranted.

Another explanation for the observed negative association between TSPO and BMI could be related to the increased level of endogenous ligands such as cholesterol and porphyrins [46], for which TSPO has high affinity. Obese subjects often have higher levels of serum cholesterol [47], which could induce competition between TSPO ligand and cholesterol between available TSPO binding sites. This hypothesis is supported by a previous study, where higher plasma cholesterol levels were shown to be associated with lower whole brain $\left[{ }^{11} \mathrm{C}\right] \mathrm{PBR} 28 \mathrm{~V}_{\mathrm{T}}$ in healthy control subjects and alcohol use disorder patients [48]. Cholesterol medication such as statins are expected to affect serum cholesterol level. However, excluding these individuals $(n=11)$ from the analysis did not change our results. Taken together, further studies on the role of TSPO and neuroinflammation in obesity are warranted to address these questions.

Our study includes some limitations. Although the subjects are imaged using the same PET scanner with similar scanning protocols, the data are acquired in different institutes with significantly different plasma parent fractions and age and BMI distributions between the study subjects. In addition, the scanner hardware and software differences, as well as differences in image analysis pipelines might increase the data variability and even influence the results on TSPO availability-associations. However, because of the nested structure of the data, we strived to control for the possible centre differences statistically by modelling a random intercept for each centre. Additionally, although all subjects were categorized as healthy controls, there are several confounding factors, which might contribute to the TSPO availability. Several older subjects were using statins to control cholesterol levels, which could theoretically affect TSPO availability, either by modulating the peripheral inflammation process or by interfering with TSPOs role in the cholesterol transport. Also, smokers have been reported to have decreased TSPO availability [49], which could contribute also to our results, as the smoking status of several subjects at the time of PET acquisition was unknown. Importantly, the main analysis results remained the same after excluding the subjects who were using cholesterol lowering or hormonal replacement medication, or whose current smoking status was positive. Thus, it is less likely that these subjects have significant effects on our age, BMI and sex findings. Recently, a paralogous Tspo gene was identified [50]. However, it is currently unknown if $\left[{ }^{11} \mathrm{C}\right] \mathrm{PBR} 28$ or any other TPSO radioligand would be sensitive to both TSPO isoforms [51] and if this isoform sensitivity affects in vivo TSPO PET measurements.

In summary, we show a positive effect of ageing on TSPO, partly replicating a previous study using $\left[{ }^{11} \mathrm{C}\right] \mathrm{PBR} 28$. In addition, we found that TSPO levels were associated with sex and BMI, which has not been demonstrated previously. In addition to identifying important confounding factors for clinical TSPO studies, the present study shows how a multicentre collaboration can mitigate the problem of small sample sizes in PET research, providing increased power to detect clinically relevant effects. Future efforts should aim at facilitating sharing of raw data, in terms of both technical and legal aspects, which would allow for increased harmonization of multi-centre analyses.

Acknowledgements We would like to thank all study participants and the staff at the Karolinska Institutet PET Centre, Turku PET Centre and Yale University PET Center for their assistance. We thank also Tomi Karjalainen for help in the analysis of Turku data.

Members of the HRRT $\left[{ }^{11} \mathrm{C}\right]$ PBR28 study group:

Jouni Tuisku ${ }^{1}$, Pontus Plavén-Sigray ${ }^{2}$, Edward C. Gaiser ${ }^{4,5}$, Laura Airas $^{1,6}$, Haidar Al-Abdulrasul ${ }^{1}$, Anna Brück ${ }^{1,6}$, Richard E. Carson ${ }^{4}$, Ming-Kai Chen ${ }^{4}$, Karin Collste ${ }^{2}$, Kelly P. Cosgrove ${ }^{4,5}$, Laura Ekblad ${ }^{1}$, Irina Esterlis ${ }^{5}$, Lars Farde ${ }^{2,7}$, Anton Forsberg ${ }^{2}$, Jean-Dominique Gallezot $^{4}$, Christer Halldin ${ }^{2}$, Semi Helin ${ }^{1}$, Ansel Hillmer ${ }^{4,5}$, Yiyun Huang ${ }^{4}$, Caroline O. Höglund ${ }^{2,9,10}$, Jarkko Johansson ${ }^{1}$, Aurelija Jucaite $^{2,7}$, Eva Kosek ${ }^{8}$, Jon Lampa ${ }^{9}$, Mats Lekander ${ }^{3,8}$, Noora Lindgren ${ }^{1}$, Päivi Marjamäki ${ }^{1}$, Nabeel Nabulsi ${ }^{4}$, Brian Pittman ${ }^{5}$, Eero Rissanen $^{1,6}$, Christine M. Sandiego ${ }^{4,5}$, Per Stenkrona ${ }^{2,}$ Marcus Sucksdorff $^{1,6}$, Andrea Varrone ${ }^{2,3}$, Juha Rinne ${ }^{1,6}$, David Matuskey ${ }^{4,5}$, Simon Cervenka ${ }^{2}$.

${ }^{I}$ Turku PET Centre, University of Turku, Turku, Finland.

${ }^{2}$ Centre for Psychiatry Research, Department of Clinical Neuroscience, Karolinska Institutet and Stockholm County, Stockholm, Sweden.

${ }^{3}$ Stress Research Institute, Department of Psychology, Stockholm University.

${ }^{4}$ PET Center, Department of Radiology and Biomedical Imaging, Yale University, New Haven, CT 06520.

${ }^{5}$ Department of Psychiatry, Yale University, New Haven, CT 06511.

${ }^{6}$ Division of Clinical Neurosciences, Turku University Hospital, Turku, Finland.

${ }^{7}$ PET Science Centre, Precision Medicine and Genomics, IMED Biotech Unit, AstraZeneca, Karolinska Institutet, Sweden.

${ }^{8}$ Department of Clinical Neuroscience, Karolinska Institutet, Stockholm, Sweden.

${ }^{9}$ Department of Medicine and Center for Molecular Medicine, Karolinska Institutet, Karolinska University Hospital, Stockholm, Sweden.

${ }^{10}$ Department of Physiology and Pharmacology, Karolinska Institutet, Stockholm, Sweden.

Authors' contributions DM, JR, SC supervised and designed the study; PSS designed the statistical model; JT and PPS performed the statistical analysis of all data; BP contributed to the statistical analysis of the data; $\mathrm{AF}$ and PPS performed the KI data processing at KI; JT performed the Turku data processing; EK and ML performed the subject recruitment for PET scanning at KI; LF, and AV participated in the generation and analysis of the [11C]PBR28 data at KI; LA, HAA, AB, LE, NL and MS performed the subject recruitment and PET scanning at Turku; REC, $\mathrm{MKC}, \mathrm{KPC}$, IE, JDG, AH, YH and CMS participated in generation and 
analysis of the [11C]PBR28 data at Yale; $\mathrm{CH}$ performed the radiochemistry at KI; SH performed the radiochemistry at Turku; NN supervised Yale PET radiochemistry; PM performed the metabolite analysis for Turku data; JT, DM, JR, PPS and SC drafted the manuscript; ECG, LA, LE, SH, ML, ER participated in manuscript writing; All authors interpreted the results, critically revised the article, and approved of the final version for publication.

Funding Open access funding provided by University of Turku (UTU) including Turku University Central Hospital. PET studies at KI were funded by grants from the Swedish Science Council. PET studies at Turku PET Centre were funded by grants from the Sigrid Juselius Foundation, the Pro Humanitate Foundation, the Finnish Cultural Foundation, the Academy of Finland (\#310962), Governmental Clinical Grants (VTR) and The Finnish Parkinson Foundation. PET studies at Yale were funded by grants from the Nancy Taylor Foundation and SNMMI molecular imaging research grant for junior faculty. JT is supported by the Alfred Kordelin Foundation, the Instrumentarium Science Foundation, the Orion research Foundation, the Paulo Foundation, the Päivikki and Sakari Sohlberg Foundation and the Turku University Hospital Foundation. SC is supported by Swedish Research Council (Grant No. 523-2014-3467). MKC is supported by Eli Lilly and company grant. NL was supported by the Finnish Cultural Foundation, Yrjö Jahnsson Foundation, Turku University Foundation, and Finnish Brain Foundation. KC is supported by the U.S. Department of Veterans Affairs National Center for posttraumatic stress disorder. ML is supported by the Hedlund Foundation, Swedish Heart-Lung Foundation, Swedish Asthma and Allergy Association and the Swedish Research Council. HA is supported by the Finnish Parkinson Foundation. LA is supported by the Academy of Finland and the Sigrid Juselius Foundation.

\section{Compliance with ethical standards}

Ethical approval All procedures performed in studies involving human participants were in accordance with the ethical standards of the institutional and/or national research committee and with the principles of the 1964 Declaration of Helsinki and its later amendments or comparable ethical standards.

Informed consent Informed consent was obtained from all individual participants included in the study.

Conflict of interest LF and AJ are employees of Astrazeneca. All other authors declare that they have no conflict of interest.

Open Access This article is distributed under the terms of the Creative Commons Attribution 4.0 International License (http:// creativecommons.org/licenses/by/4.0/), which permits unrestricted use, distribution, and reproduction in any medium, provided you give appropriate credit to the original author(s) and the source, provide a link to the Creative Commons license, and indicate if changes were made.

\section{References}

1. Notter T, Coughlin JM, Sawa A, et al. Reconceptualization of translocator protein as a biomarker of neuroinflammation in psychiatry. Mol Psychiatry. 2018;23:36-47.

2. Cosenza-Nashat M, Zhao ML, Suh HS, et al. Expression of the translocator protein of $18 \mathrm{kDa}$ by microglia, macrophages and astrocytes based on immunohistochemical localization in abnormal human brain. Neuropathol Appl Neurobiol. 2009;35:306-28.
3. Owen DR, Guo Q, Kalk NJ, et al. Determination of [(11)C]PBR28 binding potential in vivo: a first human TSPO blocking study. J Cereb Blood Flow Metab. 2014;34:989-94.

4. Kobayashi M, Jiang T, Telu S, et al. ${ }^{11}$ C-DPA-713 has much greater specific binding to translocator protein $18 \mathrm{kDa}$ (TSPO) in human brain than ${ }^{11} \mathrm{C}-(R)$-PK11195. J Cereb Blood Flow Metab. 2018;38:393-403.

5. Doble A, Malgouris C, Daniel M, et al. Labelling of peripheral-type benzodiazepine binding sites in human brain with [3H]PK 11195: Anatomical and subcellular distribution. Brain Res Bull. 1987;18: 49-61.

6. Cumming P, Burgher B, Patkar O, et al. Sifting through the surfeit of neuroinflammation tracers. J Cereb Blood Flow Metab. 2018;38: 204-24.

7. Owen DR, Yeo AJ, Gunn RN, et al. An 18-kDa Translocator translocator protein (TSPO) polymorphism explains differences in binding affinity of the PET radioligand PBR28. J Cereb Blood Flow Metab. 2012;32:1-5.

8. Collste K, Forsberg A, Varrone A, et al. Test-retest reproducibility of [11C]PBR28 binding to TSPO in healthy control subjects. Eur J Nucl Med Mol Imaging. 2016;43:173-83.

9. Papadopoulos V, Baraldi M, Guilarte TR, et al. Translocator protein (18kDa): new nomenclature for the peripheral-type benzodiazepine receptor based on its structure and molecular function. Trends Pharmacol Sci. 2006;27:402-9.

10. Velarde MC. Mitochondrial and sex steroid hormone crosstalk during aging. Longev Heal. 2014;3:2.

11. Cagnin A, Brooks DJ, Kennedy AM, et al. In-vivo measurement of activated microglia in dementia. Lancet. 2001;358:461-7.

12. Schuitemaker A, van der Doef TF, Boellaard R, et al. Microglial activation in healthy aging. Neurobiol Aging. 2012;33:1067-72.

13. Paul S, Gallagher E, Liow J-S, et al. Building a database for brain $18 \mathrm{kDa}$ translocator protein imaged using [ ${ }^{11} \mathrm{C}$ ]PBR28 in healthy subjects. J Cereb Blood Flow Metab. 2018:0271678X1877125.

14. Rissanen E, Tuisku J, Vahlberg T, et al. Microglial activation, white matter tract damage, and disability in MS. Neurol Neuroimmunol Neuroinflamm. 2018;5:e443.

15. Debruyne JC, Versijpt J, Van Laere KJ, et al. PET visualization of microglia in multiple sclerosis patients using [11C]PK11195. Eur J Neurol. 2003;10:257-64.

16. Suridjan I, Rusjan PM, Voineskos AN, et al. Neuroinflammation in healthy aging: A PET study using a novel translocator Protein $18 \mathrm{kDa}$ (TSPO) radioligand, [18F]-FEPPA. Neuroimage. 2014;84: 868-75.

17. Yasuno F, Ota M, Kosaka J, et al. Increased binding of peripheral benzodiazepine receptor in Alzheimer's disease measured by positron emission tomography with [11C]DAA1106. Biol Psychiatry. 2008;64:835-41.

18. Collste K, Plavén-Sigray P, Fatouros-Bergman H, et al. Lower levels of the glial cell marker TSPO in drug-naive first-episode psychosis patients as measured using PET and [11C]PBR28. Mol Psychiatry. 2017;22:850-6.

19. Hannestad J, DellaGioia N, Gallezot J-D, et al. The neuroinflammation marker translocator protein is not elevated in individuals with mild-to-moderate depression: a $\left[{ }^{11} \mathrm{C}\right]$ PBR28 PET study. Brain Behav Immun. 2013;33:131-8.

20. Jucaite A, Svenningsson P, Rinne JO, et al. Effect of the myeloperoxidase inhibitor AZD3241 on microglia: a PET study in Parkinson's disease. Brain. 2015;138:2687-700.

21. Tzourio-Mazoyer N, Landeau B, Papathanassiou D, et al. Automated anatomical labeling of activations in SPM using a macroscopic anatomical parcellation of the MNI MRI single-subject brain. Neuroimage. 2002;15:273-89.

22. Ichise M, Toyama H, Innis RB, et al. Strategies to improve neuroreceptor parameter estimation by linear regression analysis. J Cereb Blood Flow Metab. 2002;22:1271-81. 
23. Matheson GJ, Plavén-Sigray P, Forsberg A, et al. Assessment of simplified ratio-based approaches for quantification of PET [11C]PBR28 data. EJNMMI Res. 2017;7:58.

24. Damani MR, Zhao L, Fontainhas AM, et al. Age-related alterations in the dynamic behavior of microglia. Aging Cell. 2011;10:263-76.

25. DiPatre PL, Gelman BB. Microglial cell activation in aging and Alzheimer disease: partial linkage with neurofibrillary tangle burden in the hippocampus. J Neuropathol Exp Neurol. 1997;56:1439.

26. Miller KR, Streit WJ. The effects of aging, injury and disease on microglial function: a case for cellular senescence. Neuron Glia Biol. 2007;3:245-53.

27. Sheng JG, Mrak RE, Griffin WS. Enlarged and phagocytic, but not primed, interleukin-1 alpha-immunoreactive microglia increase with age in normal human brain. Acta Neuropathol. 1998;95:22934.

28. Michell-Robinson MA, Touil H, Healy LM, et al. Roles of microglia in brain development, tissue maintenance and repair. Brain. 2015;138:1138-59.

29. Godbout JP, Johnson RW. Age and neuroinflammation: a lifetime of psychoneuroimmune consequences. Neurol Clin. 2006;24:52138.

30. Henry CJ, Huang Y, Wynne AM, et al. Peripheral lipopolysaccharide (LPS) challenge promotes microglial hyperactivity in aged mice that is associated with exaggerated induction of both proinflammatory IL- $1 \beta$ and anti- inflammatory IL-10 cytokines. Brain Behav Immun. 2009;23:309-17.

31. Njie G, Boelen E, Stassen FR, et al. Ex vivo cultures of microglia from young and aged rodent brain reveal age-related changes in microglial function. Neurobiol Aging. 2012;33(1):195.e112.https://doi.org/10.1016/j.neurobiolaging.2010.05.008.

32. Sierra A, Gottfried-Blackmore AC, McEwen BS, et al. Microglia derived from aging mice exhibit an altered inflammatory profile. Glia. 2007;55:412-24.

33. Schwarz JM, Sholar PW, Bilbo SD. Sex differences in microglial colonization of the developing rat brain. J Neurochem. 2012;120: 948-63.

34. Villa A, Gelosa P, Castiglioni L, et al. Sex-specific features of microglia from adult mice. Cell Rep. 2018;23:3501-11.

35. Nissen JC. Microglial function across the spectrum of age and gender. Int J Mol Sci. 2017;18(3).https://doi.org/10.3390/ ijms 18030561 .

36. Puy L, MacLusky NJ, Becker L, et al. Immunocytochemical detection of androgen receptor in human temporal cortex characterization and application of polyclonal androgen receptor antibodies in frozen and paraffin-embedded tissues. J Steroid Biochem Mol Biol. 1995;55:197-209.

37. Uchoa MF, Moser VA, Pike CJ. Interactions between inflammation, sex steroids, and Alzheimer's disease risk factors. Front Neuroendocrinol. 2016;43:60-82.

38. Barreto G, Veiga S, Azcoitia I, et al. Testosterone decreases reactive astroglia and reactive microglia after brain injury in male rats: role of its metabolites, oestradiol and dihydrotestosterone. Eur J Neurosci. 2007;25:3039-46.

39. Villa A, Vegeto E, Poletti A, et al. Estrogens, neuroinflammation, and neurodegeneration. Endocr Rev. 2016;37:372-402.

40. Erion JR, Wosiski-Kuhn M, Dey A, et al. Obesity elicits interleukin 1-mediated deficits in hippocampal synaptic plasticity. J Neurosci. 2014;34:2618-31.

41. Buckman LB, Thompson MM, Lippert RN, et al. Evidence for a novel functional role of astrocytes in the acute homeostatic response to high-fat diet intake in mice. Mol Metab. 2015;4:58-63.

42. Sobesky JL, Barrientos RM, De May HS, et al. High-fat diet consumption disrupts memory and primes elevations in hippocampal IL- $1 \beta$, an effect that can be prevented with dietary reversal or IL-1 receptor antagonism. Brain Behav Immun. 2014;42:22-32.

43. Hao S, Dey A, Yu X, et al. Dietary obesity reversibly induces synaptic stripping by microglia and impairs hippocampal plasticity. Brain Behav Immun. 2016;51:230-9.

44. Li J, Papadopoulos V. Translocator protein $(18 \mathrm{kDa})$ as a pharmacological target in adipocytes to regulate glucose homeostasis. Biochem Pharmacol. 2015;97:99-110.

45. Thompson MM, Manning HC, Ellacott KLJ. Translocator protein $18 \mathrm{kDa}$ (TSPO) is regulated in white and brown adipose tissue by obesity. PLoS One. 2013;8:e79980.

46. Kanegawa N, Collste K, Forsberg A, et al. In vivo evidence of a functional association between immune cells in blood and brain in healthy human subjects. Brain Behav Immun. 2016;54:149-57.

47. Guo Y, Kalathur RC, Liu Q, et al. Protein structure. Structure and activity of tryptophan-rich TSPO proteins. Science. 2015;347:5515.

48. Després JP, Moorjani S, Lupien PJ, et al. Regional distribution of body fat, plasma lipoproteins, and cardiovascular disease. Arteriosclerosis. 1990;10:497-511.

49. Kim SW, Wiers CE, Tyler R, et al. Influence of alcoholism and cholesterol on TSPO binding in brain: PET [11 C]PBR28 studies in humans and rodents. Neuropsychopharmacology. 2018;43(9): 1832-1839.https://doi.org/10.1038/s41386-018-0085-x.

50. Brody AL, Hubert R, Enoki R, et al. Effect of cigarette smoking on a marker for neuroinflammation: a [11C]DAA1106 positron emission tomography study. Neuropsychopharmacology. 2017;42: 1630-9.

51. Fan J, Rone MB, Papadopoulos V. Translocator protein 2 is involved in cholesterol redistribution during erythropoiesis. J Biol Chem. 2009;284:30484-97.

52. Iatmanen-Harbi S, lucile S, Papadopoulos V, et al. Characterization of the high-affinity drug ligand binding site of mouse recombinant TSPO. Int J Mol Sci. 2019;20:1444.

Publisher's note Springer Nature remains neutral with regard to jurisdictional claims in published maps and institutional affiliations. 


\section{Affiliations}

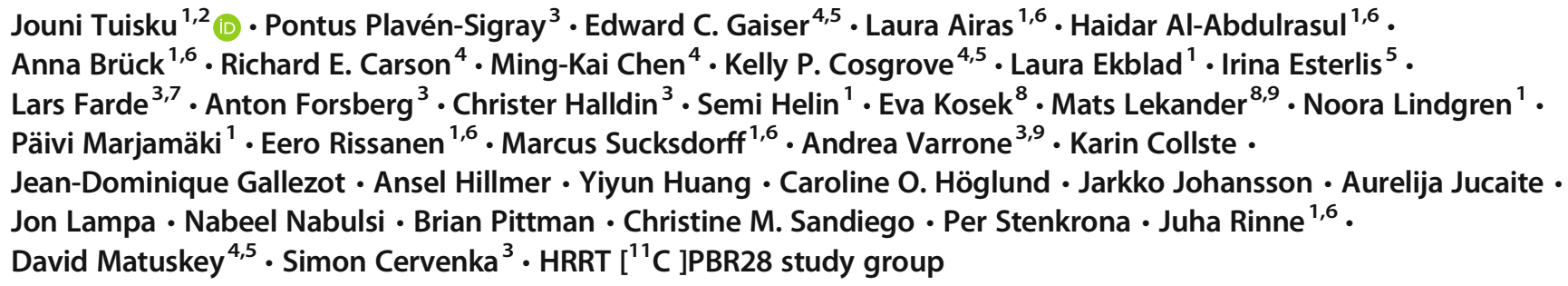

Pontus Plavén-Sigray

Pontus.Plaven-Sigray@ki.se

Edward C. Gaiser

edward.gaiser@yale.edu

Laura Airas

laura.airas@utu.fi

Haidar Al-Abdulrasul

Haidar.Al-Abdulrasul@tyks.fi

Anna Brück

Anna.Bruck@tyks.fi

Richard E. Carson

richard.carson@yale.edu

Ming-Kai Chen

ming-kai.chen@yale.edu

Kelly P. Cosgrove

kelly.cosgrove@yale.edu

Laura Ekblad

llekbl@utu.fi

Irina Esterlis

Irina.Esterlis@yale.edu

Lars Farde

lars.farde@ki.se

Anton Forsberg

anton.forsberg@ki.se

1 Turku PET Centre, University of Turku, Turku, Finland

2 Turku PET Centre, c/o Turku University Hospital, Po Box 52, 20521 Turku, Finland

3 Centre for Psychiatry Research, Department of Clinical Neuroscience, Karolinska Institutet and Stockholm County, Stockholm, Sweden

4 PET Center, Department of Radiology and Biomedical Imaging, Yale University, New Haven, CT 06520, USA

5 Department of Psychiatry, Yale University, New Haven, CT 06511, USA

\author{
Christer Halldin \\ christer.halldin@ki.se \\ Semi Helin \\ Semi.Helin@abo.fi \\ Eva Kosek \\ eva.kosek@ki.se \\ Mats Lekander \\ mats.lekander@ki.se \\ Noora Lindgren \\ nhsalm@utu.fi \\ Päivi Marjamäki \\ pamarja@utu.fi \\ Eero Rissanen \\ eerris@utu.fi \\ Marcus Sucksdorff \\ marsuc@utu.fi \\ Andrea Varrone \\ andrea.varrone@ki.se \\ Juha Rinne \\ Juha.Rinne@tyks.fi \\ David Matuskey \\ david.matuskey@yale.edu \\ Simon Cervenka \\ Simon.Cervenka@ki.se
}

6 Division of Clinical Neurosciences, Turku University Hospital, Turku, Finland

7 PET Science Centre, Precision Medicine and Genomics, IMED Biotech Unit, AstraZeneca, Karolinska Institutet, Solna, Sweden

8 Department of Clinical Neuroscience, Karolinska Institutet, Stockholm, Sweden

9 Stress Research Institute, Department of Psychology, Stockholm University, Stockholm, Sweden 